\title{
The Role of Through-Bond Stereoelectronic Effects in the Reactivity of 3-Azabicyclo[3.3.1]nonanes
}

\author{
Croix J. Laconsay, Tyler C. Rho, and Dean J. Tantillo* \\ Department of Chemistry, University of California—Davis, CA 95616 \\ email: djtantillo@ucdavis.edu
}

KEYWORDS. remote stereoelectronic effects; through-bond interactions; hetereolytic fragmentation; isodesmic reactions; hydration propensity; basicity

ABSTRACT. Hyperconjugation/conjugation through-bond stereoelectronic effects were studied with density functional theory (DFT) in the context of 3-azabicyclo[3.3.1]nonanes to unravel puzzling differences in reactivity between a vinylogous chloride (4) and a vinylogous ester (5). These compounds - whose structures differ only by one substituent—were found to display strikingly different reactivities in hydrochloric acid by Risch and co-workers (J. Am. Chem. Soc. 1991, 113, 9411-9412). Computational analyses of substituent effects, noncovalent interactions, natural bond orbitals, isodesmic reactions, and hydration propensities lead to a model for which the role of remote, through-bond stereoelectronic effects is key to explaining $\mathbf{4}$ and $\mathbf{5}$ 's diverging reactivity. 


\section{INTRODUCTION}

Bond, through-bond. ${ }^{1}$ Interactions in chemistry can occur through-space and/or throughbond, ${ }^{2-4}$ both of which have been the subject of intense research in theoretical chemistry ${ }^{2,3}$ and photoelectron spectroscopy. ${ }^{5-10}$ These interactions can affect reactivity/selectivity of molecules because their manifestations are net stabilizing or destabilizing and depend on molecular geometry, hence stereoelectronic. ${ }^{11-16}$ For example, many investigations have been sparked by 1azaadamantane structures (e.g., Scheme 1, 1) because of its unique, conformationally constrained architecture, ${ }^{17-19}$ rich in hyperconjugation. ${ }^{11,20,21}$ Additionally, 1-azaadamantanes (and derivatives like 1-azaadamantanones) have been synthesized ${ }^{22,23}$ and studied in the past for their tendency to undergo Grob fragmentations,${ }^{24}$ for their intramolecular through-bond ${ }^{16}$ and charge transfer interactions, ${ }^{25}$ and for measuring electron spin distribution through a $\sigma$-skeleton by ${ }^{13} \mathrm{C}$ contact shifts in NMR studies. ${ }^{26}$ We developed an interest in 1-azaadamantanone structures (e.g., 1, Scheme 1) and their 3-azabicyclo[3.3.1]nonane fragmentation products (e.g., 4 and 5, Scheme 1) for their visually appealing ${ }^{27}$ and unusual structures, which hold functional groups of varying nucleophilicity and electrophilicity in particular orientations. 3-Azabicyclo[3.3.1]nonanes ${ }^{28}$ are of interest for their pharmaceutical applications ${ }^{29,30}$ and use in molecular recognition chemistry. ${ }^{31,32}$ The 3-azabicyclo[3.3.1]nonane architecture also arises in Aristotelia alkaloid natural products (e.g., hobartine and aristoquinoline). ${ }^{33,34}$

Heterolytic fragmentation reactions (e.g., $\mathbf{2} \rightarrow \mathbf{3}$ ) can be useful strategic tools in organic synthesis. ${ }^{35-41}$ When applied successfully, fragmentation reactions can effect ringexpansion $^{42,43}$ — see, for example, the total synthesis of vinigrol ${ }^{44}$ Examples in which fragmentation reactions have been useful continue to emerge in the literature (e.g., in bioorthogonal self-immolative linkers ${ }^{45}$ and we suspect their utility will only continue to 
expand. Nevertheless, sparse mechanistic information hinders their broad use. Somewhat cryptic aspects of fragmentations that computational chemistry is uniquely positioned to address include: (1) directing competing divergent mechanisms to control product outcomes, ${ }^{46}$ (2) connection between the atom-length of a fragmentation and its concertedness, and (3) tendencies of particular groups to sequentially fragment (in stepwise or concerted but asynchronous pathways). ${ }^{24}$ Basic research ${ }^{47}$ into simple theoretical model systems that investigate the roles of stereoelectronic interactions in fragmentations, and intermediates/products born out of them, would therefore be valuable for future investigations of heterolytic fragmentations. To this aim, we consider here through-bond effects in a 3-azabicyclo[3.3.1]nonane product borne out of a fragmentation reaction $^{48}$ — orbital effects that could have promoted its formation and that influence its fate.

Compound 1, reported by Risch's group, piqued our interest because it was reported that 1 fragments to a "stable" intermediate, $4{ }^{48}$ Here, stable refers to kinetic stability (as opposed to thermodynamic stability) with respect to hydrolysis. Specifically, diketone $\mathbf{1}$ yields $\mathbf{2}$ when refluxed in thionyl chloride. Molecule 2 then fragments to a 3-azabicyclo[3.3.1]nonane derivative, 4, upon workup [(1) aqueous ammonia, 25\%; (2) methanol/ $\mathrm{NaOH}$; (3) concentrated hydrochloric acid]. ${ }^{48}$ An alternative fragmentation of $\mathbf{2}$ could generate $\beta, \gamma$-unsaturated ketone $\mathbf{6}$, but the authors do not observe 6. Our calculations are consistent with this result: fragmentation to $\mathbf{6}$ is kinetically and thermodynamically less favorable than fragmentation to $\mathbf{3}\left(\Delta \mathrm{G}^{\dagger}=24.3 \mathrm{kcal}\right.$ $\mathrm{mol}^{-1}, \Delta \mathrm{G}=16.4 \mathrm{kcal} \mathrm{mol}^{-1}$ to 6 versus $\Delta \mathrm{G}^{*}=22.2 \mathrm{kcal} \mathrm{mol}^{-1}, \Delta \mathrm{G}=10.2 \mathrm{kcal} \mathrm{mol}^{-1}$ to 3 ; see Supporting Information (SI), Figure S1) Notably, treatment of 4 with $\mathrm{CH}_{3} \mathrm{O}^{-}$was reported to give structure 5, which "immediately reacts with hydrochloric acid at room temperature" 48 to form $\mathbf{1}$, presumably via initial hydrolysis to the vinylogous acid. 
Scheme 1. Unusual reorganization of 3-azabicyclo[3.3.1]nonanes through heterolytic fragmentation.

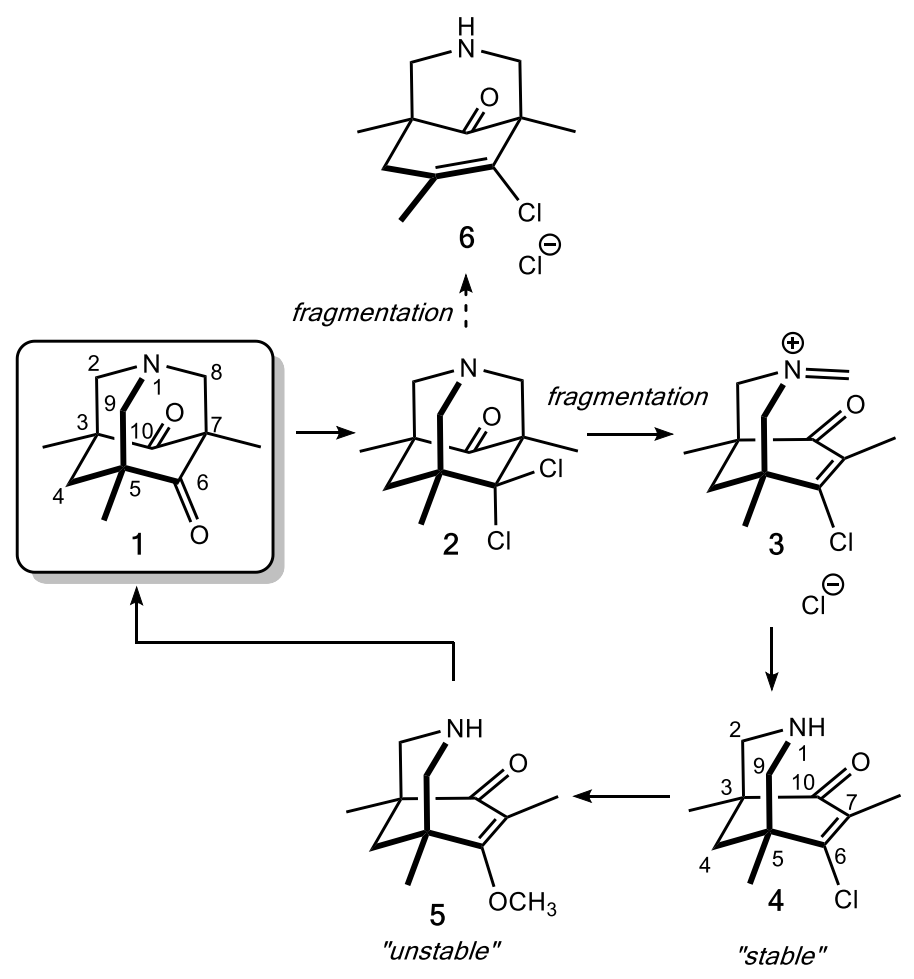

The Puzzle

Herein, we use density functional theory (DFT) calculations to investigate why $\mathbf{5}$, and not 4, react in acid. While $\mathbf{4}$ is a vinylogous chloride, $\mathbf{5}$ is a vinylogous ester. Why $\mathbf{4}$ and $\mathbf{5}$ would react so differently in acid is not obviously clear, especially since one would expect an acid chloride to be more susceptible to hydrolysis than an ester. One possible answer is that swapping a chlorine atom at C6 in $\mathbf{4}$ to a methoxy group in $\mathbf{5}$ results in changes in intramolecular orbital interactions in $\mathbf{4}$ that are not present, or are of different strengths, in $\mathbf{5}$. Here we assess how interactions between the amine and $\alpha, \beta$-unsaturated carbonyl substructures influence reactivity, 
both through-space and through-bond. ${ }^{49}$ Our results reveal that the divergent reactivity can be tied to through-bond orbital effects, which are scaffolded by the 1-azaadamantane cage.

\section{METHODS}

Density functional theory calculations were carried out using Gaussian $09 .{ }^{50}$ The Minnesota hybrid functional, M06-2X, ${ }^{51}$ was used for geometry optimization and frequency analysis for calculation of Gibbs free energies, as it has been shown to work well for systems involving hyperconjugation and minimizes error associated with extensive electron delocalization. ${ }^{52,53}$ A triple- $\zeta$ Pople basis set, $6-311+\mathrm{G}(\mathrm{d}, \mathrm{p})$, was used with diffuse and polarization functions for all calculations. Intrinsic reaction coordinate (IRC) calculations were carried out to find the minimum energy pathways downhill in energy and each transition state structure's flanking minima. ${ }^{54-56}$ All computed structures (including coordinates) can be found on the ioChem-BD repository ${ }^{57}$ at the following DOI: https://doi.org/10.19061/iochem-bd-6-120. Energies, file names, and lowest frequencies associated with each structure can also be found in the SI.

Basicities were computed by using the proton transfer equilibrium equation (1) and a formula based on electronic energies (2) ${ }^{58}$ Relative basicity values $(B)$ obtained from this method establish a reasonable sense of relative basicity for various R-substituted fragmented products (FPs).

$$
\begin{gathered}
A+H^{+} \rightarrow A H^{+} \\
B=E\left(F P H^{+}+H_{2} O\right)-E\left(F P+H_{3} O^{+}\right)
\end{gathered}
$$


Noncovalent interaction (NCI) plots ${ }^{59}$ were generated using Multiwfn version 3.7 with a medium quality grid. ${ }^{60}$ Natural bond orbital (NBO) analysis (version 3.1) was carried out in Gaussian 09 to obtain second-order perturbation NBO energies. ${ }^{61,62}$ Hydration propensities of our substrates were calculated using electronic energies $(E)$ in a formula based on thermodynamic data ${ }^{63}$ — hydration data may also be determined kinetically ${ }^{64,65}$ — with the following equations ( 3 and 4 ), where $E$ is the non-hydrated species, and $E_{\text {hyd }}$ is the hydrated species:

$$
\begin{aligned}
& \Delta E_{r x n}=E_{h y d}-\left(E+E_{H_{2} O}\right) \\
& l n K_{h y d}=-\left(\frac{\Delta E_{r x n}}{R T}\right)
\end{aligned}
$$

\section{RESULTS AND DISCUSSION}

\section{Noncovalent Interactions (NCI) Analysis}

Structures $\mathbf{4}$ and $\mathbf{5}$ are unique in that the amine and $\pi$-system are close enough that a through-space interaction could be relevant between the lone pair $\left(\mathrm{n}_{N}\right)$ of the nitrogen and the $\pi$ system (i.e., $\mathrm{n}_{\mathrm{N}} \leftrightarrow \pi^{*} \mathrm{C}=\mathrm{C}$ or $\mathrm{n}_{\mathrm{N}} \leftrightarrow \pi^{*} \mathrm{C}=\mathrm{O}$ ) or between the $\mathrm{N}-\mathrm{H}$ bond and the $\pi$-system. If throughspace interactions are operative, then a noncovalent interaction (NCI) analysis should reveal any difference in through-space interactions that exist between 4 and $\mathbf{5}$. NCI analysis is a useful tool for qualitatively visualizing intra- and intermolecular noncovalent interactions within (or between) molecules. ${ }^{59}$ The use of NCI analyses in computational organic chemistry studies has become increasingly important to characterize, visualize, and rationalize the role of weak, noncovalent interactions in mechanistic models. ${ }^{66-68}$ Colors in NCI plots indicate the type of interaction: blue indicates strong attraction on one extreme, and red indicates strong repulsion on 
the other; green lies in the middle of the two extremes and indicates weak interactions (e.g., dispersion interactions). Figure 1 shows NCI plots for structures $\mathbf{4}$ and $\mathbf{5}$. The green in each plot indicates that weak interactions exist between the amine and the $\pi$-system, but the degree to which it changes from $\mathbf{4}$ to $\mathbf{5}$ suggests that through-space effects remain effectively the same in $\mathbf{4}$ and 5 (analogous results were obtained for the $N$-invertomers of $\mathbf{4}$ and $\mathbf{5}$ and their $N$-protonated forms; see SI for details). A through-space interaction argument alone is thus insufficient to explain Risch et al.'s observed diverging kinetic stabilities in acid. Though these plots by themselves do not definitively rule out the existence of strong through-space interactions between the amine and the enone moieties, they weigh against through-space effects having a consequential role in the fate of these structures in acid.
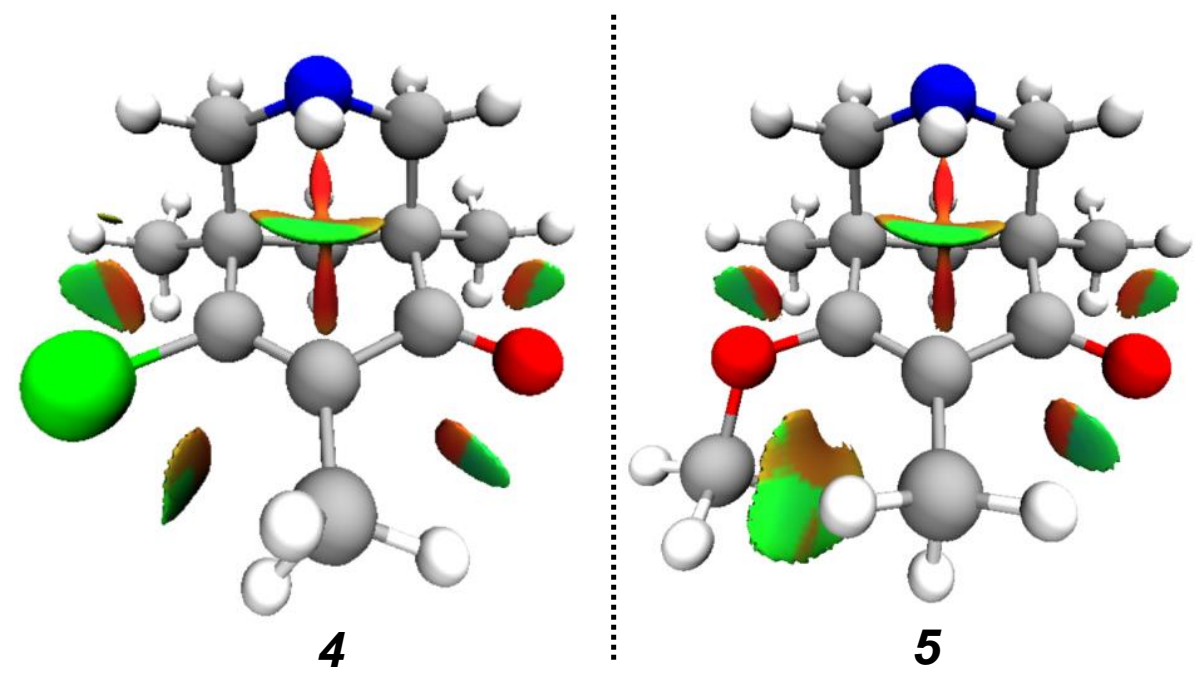

Figure 1. NCI index plots (blue, strong attraction; green, weak interaction; red, strong repulsion; isovalue $=0.5$ ) of structures 4 and 5 .

Basicity of Amine and $\pi$-system 
If, on the other hand, through-bond effects are important for the stabilities in acid of 4 and $\mathbf{5}$, we would expect to see a significant difference in computed basicity for the amine, the most basic site of $\mathbf{4}$ and $\mathbf{5}$. The strong basicity of the amine relative to other groups is reflected in our computed basicities below: in Figures 2 and 3 when $\mathrm{R}=\mathrm{H}$, the basicities of the ketone and amine are -45.7 and $-61.7 \mathrm{kcal} \mathrm{mol}^{-1}$, respectively.

Both $\mathbf{4}$ and $\mathbf{5}$ have local amine functionality and local vinylogous carbonyl derivative functionality. But these local functional groups may communicate with each other by throughbond orbital effects. If this is the case, we would expect to observe changes in amine basicity upon changing the substituent at carbon $6(\mathrm{C} 6$, Scheme 1); it would not be the first time amine basicity is influenced by remote, neighboring functionality. ${ }^{69}$ Figure 2 shows the results of our amine basicity calculations with different $\mathrm{C} 6$ substituents plotted against their Hammett $\sigma_{\text {para }}$ constants, which reflect the $\pi$ electron-donating and -withdrawing ability of R. ${ }^{70,71}$ Though the range of basicity (ca. $10 \mathrm{kcal} \mathrm{mol}^{-1}$ ) for the amine pales in comparison to the range of computed basicity for the ketone (Figure 3, ca. $\left.30 \mathrm{kcal} \mathrm{mol}^{-1}\right)$, the correlation $\left(\mathrm{R}^{2}=0.88\right)$ with $\sigma_{\text {para }}$ values suggest that the amine lone pair is sensitive to the nature of the R group, an observation consistent with a through-bond interaction. Additionally, the difference in basicity values between 4 and 5, a $\Delta \Delta \mathrm{E}=5.3 \mathrm{kcal} \mathrm{mol}^{-1}$, suggests that a $\mathrm{Cl}$ at $\mathrm{C} 6$ (4) makes the amine notably less basic than when a methoxy group is at C6 (5). 


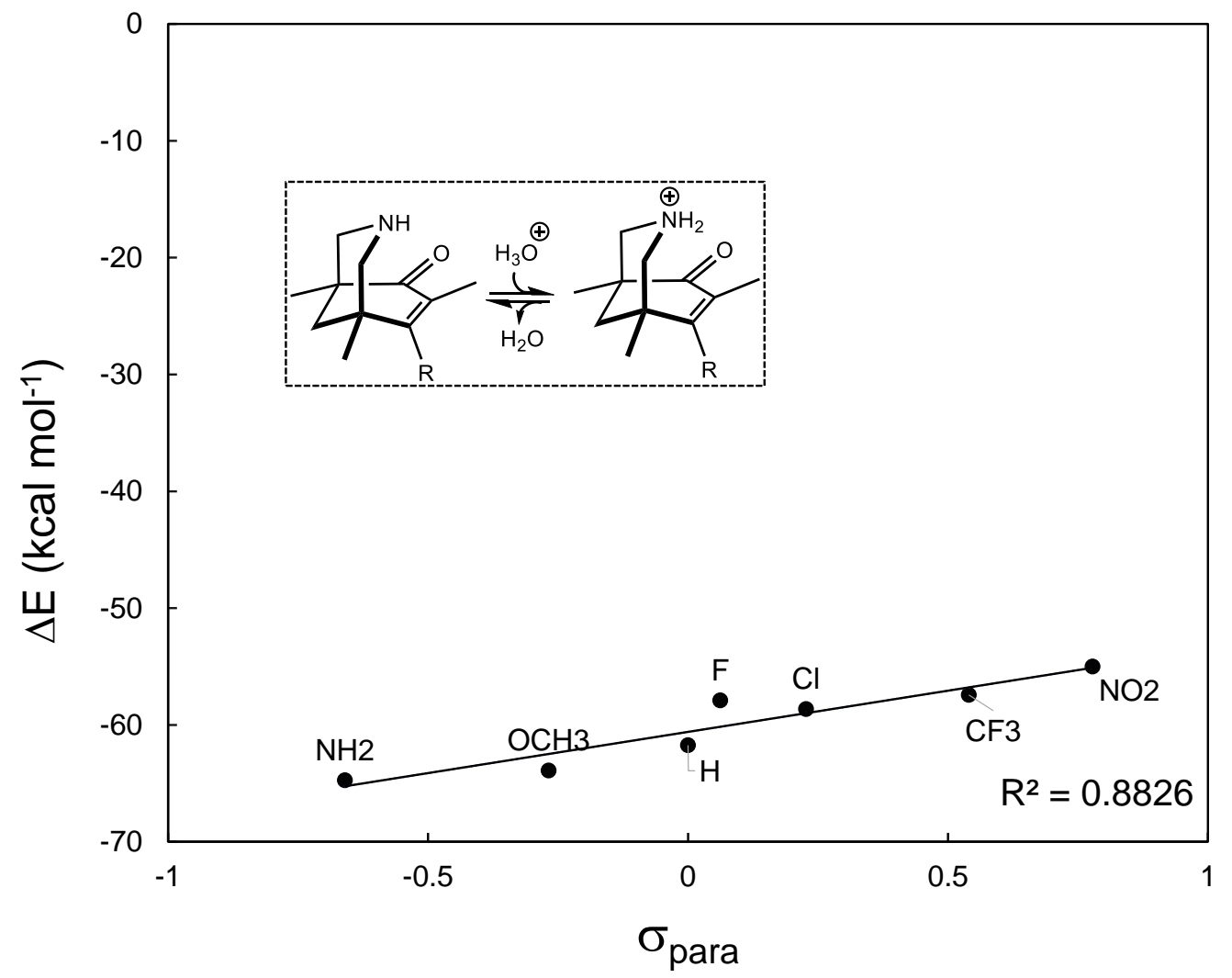

Figure 2. Relationship between R substituent $\sigma_{\text {para }}$ constant and basicity of nitrogen.

The same basicity analysis was applied to the ketone group (Figure 3). Oxygen basicities are highly correlated with the Hammett $\sigma_{\text {para }}$ value $\left(\mathrm{R}^{2}=0.97\right)$, as expected. Compound $\mathbf{5}(\mathrm{R}=$ $\left.\mathrm{OCH}_{3}\right)$ has a greater ketone oxygen basicity than that of $4(\mathrm{R}=\mathrm{Cl}): \Delta \mathrm{E}$ 's $=-55.5 \mathrm{kcal} \mathrm{mol}^{-1}$ and $44.1 \mathrm{kcal} \mathrm{mol}^{-1}$ for 5 and $\mathbf{4}$, respectively. The greater basicity of $\mathbf{5}$ likely contributes to its greater instability in acidic solution by promoting its activation as an electrophile. 


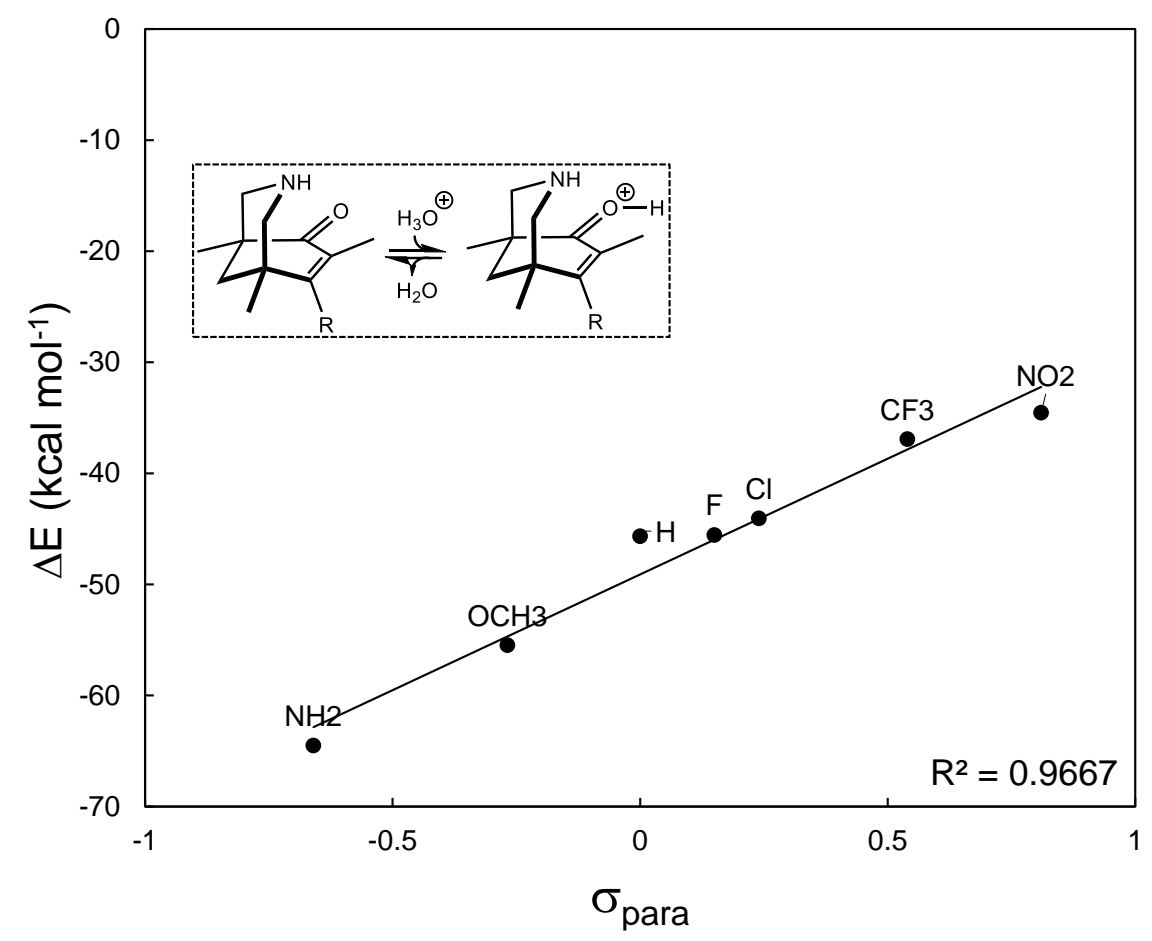

Figure 3. Relationship between R substituent $\sigma_{\text {para }}$ constant and basicity of carbonyl oxygen.

\section{It Takes Two to Tango-Hyperconjugation/Conjugation Effects}

How exactly does changing R influence the basicity of the amine? We surmised based on past literature on 1-azaadamantanones (such as $\mathbf{1}$ ), the precursor to intermediates $\mathbf{4}$ and $\mathbf{5}$, that through-bond interactions may be the major means of modulating amine basicity. ${ }^{72,73}$ Despite the nitrogen lone pair being two bonds away from the carbonyl, it still participates in an interaction resembling that of double hyperconjugation or hyperconjugation/conjugation (Scheme 2). ${ }^{74,75}$ In this particular case, the nitrogen lone pair in 1-azaadamantanones donates electron density into the $\sigma^{*}$ orbital of the adjacent $\mathrm{C}-\mathrm{C}$ bond, while simultaneously, the $\sigma$ orbital of the $\mathrm{C}-\mathrm{C}$ bond donates electron density into the $\pi^{*} \mathrm{C}=\mathrm{O}$ (Scheme $2 \mathrm{e}$ and $\left.2 \mathrm{f}\right) .{ }^{76}$ Both double hyperconjugation 
(distinct from "two-way" hyperconjugation ${ }^{77}$ ) and hyperconjugation/conjugation are examples of remote stereoelectronic effects inextricably linked by a mediating single-bond bridge, and ample examples of their effects on organic structure and reactivity are documented..$^{11,78-82}$ If intermediates $\mathbf{4}$ and $\mathbf{5}$ indeed exhibit through-bond communication between the nitrogen lone pair and the enone substructure, we would expect computed basicities to be sensitive to R's identity, and that is what is observed (Figure 2).

Scheme 2. Select types of extended hyperconjugation (see ref. 11, Ch. 8 for an in-depth discussion of remote stereoelectronic effects). a) double hyperconjugation, b) hyperconjugation/conjugation, c) negative double hyperconjugation, d-f) negative hyperconjugation/conjugation

a)

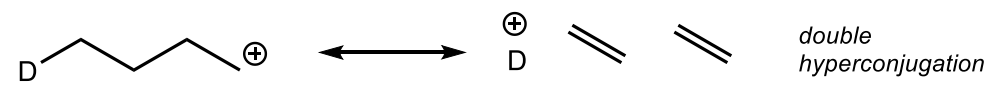

b)<smiles>C=CCC[CH2+]</smiles>

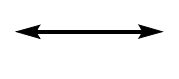<smiles>[C+]C=C</smiles><smiles>C=C</smiles>

hyperconjugation/ conjugation

c)<smiles>[Y]CCC[Hg]</smiles>

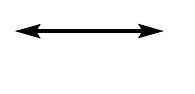<smiles>[18O]=CC=[Te]</smiles>

negative double

d)

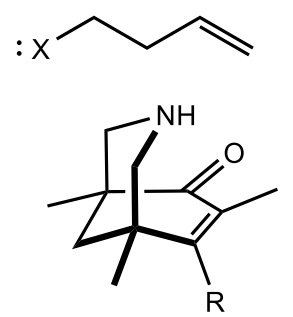<smiles>Ic1ccccc1</smiles>

f)
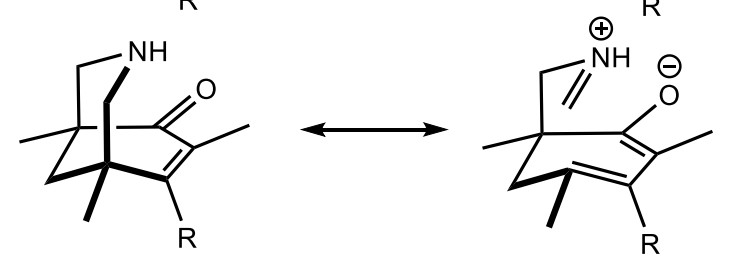

negative hyperconjugation/ conjugation (7-atom) 


\section{Natural Bond Orbital Analysis}

To further explore the possibility of through-bond effects, we computed second-order perturbation NBO energies, an analysis often used to quantify the strength of hyperconjugative donor-acceptor orbital interactions. ${ }^{20}$ Second-order perturbation NBO energy $(E(2)$, equation 5) analysis measures donor-acceptor electron delocalization that results from filled orbitals interacting with unfilled, antibonding orbitals. ${ }^{83}$ In equation (5), $n_{\sigma}$ is the population of $\sigma$ donor orbitals, $F_{\mathrm{i}, \mathrm{j}}$ is the Fock matrix element between orbitals $i$ and $j$, and $\varepsilon_{\sigma}$ and $\varepsilon_{\sigma^{*}}$ is the energies of $\sigma$ and $\sigma^{*}$ natural bond orbitals (if, for example, one is computing $E(2)$ energies between $\sigma$ and $\sigma^{*}$ orbitals).

$$
E(2)=-n_{\sigma} \frac{<\sigma|F| \sigma *>^{2}}{\varepsilon_{\sigma *}-\varepsilon_{\sigma}}=-n_{\sigma} \frac{F_{i, j}^{2}}{\Delta E} .
$$

If through-bond effects are relevant to the stability of $\mathbf{4}$ and $\mathbf{5}$, then we would expect to observe qualitative trends in $E(2) \mathrm{NBO}$ energies. Figure 4 plots $E(2) \mathrm{NBO}$ energies with Hammett $\sigma_{\text {para }}$ values. In the first case (Figure $4 a$ ), sums of $E(2)$ values associated with hyperconjugation from $n_{N} \leftrightarrow \sigma^{*}{ }_{C-C}$ of both adjacent $\mathrm{C}-\mathrm{C}$ sigma bonds are plotted against $\sigma_{\text {para }}$ values. A weak correlation $\left(R^{2}=0.66\right)$ suggests that as the electron-withdrawing ability of the $R$ substituent increases, donation into $\sigma^{*}{ }_{\text {C-C }}$ antibonding orbitals slightly increases $\left(\sim 1 \mathrm{kcal} \mathrm{mol}^{-1}\right.$ increase in $E(2))$. In other words, through-bond communication between the nitrogen lone pair and $\mathrm{R}$ is non-negligible. The sum of $E(2)$ values corresponding to $\mathrm{n}_{\mathrm{N}} \leftrightarrow \sigma_{\mathrm{C}-\mathrm{H}}^{*}$ hyperconjugation interactions (Figure $4 \mathrm{~b}$ ) are slightly better correlated $\left(\mathrm{R}^{2}=0.72\right)$ with respect to $\sigma_{\text {para }}$ values, but smaller in magnitude (at most, $7.1 \mathrm{kcal} \mathrm{mol}{ }^{-1}$ when $\mathrm{R}=\mathrm{NO}_{2}$ ) because $\sigma^{*}$-H orbitals are worse acceptors, in part, due to their poor orientation for good orbital overlap with respect to the nitrogen lone pair. No correlation between the sum of $E(2)$ values corresponding to $\sigma_{\mathrm{C}-\mathrm{C}} \leftrightarrow \pi^{*}{ }_{\mathrm{C}=\mathrm{C}}$ 
and $\sigma_{\mathrm{C}-\mathrm{C}} \leftrightarrow \pi^{*}{ }_{\mathrm{C}=\mathrm{O}}$ donor-acceptor interactions and $\sigma_{\text {para }}$ values is observed (Figure $4 \mathrm{c}$ ), To ensure our NBO analyses were reasonable, we checked whether a correlation is observed between the $\pi_{\mathrm{C}=\mathrm{C}} \leftrightarrow \pi^{*}{ }_{\mathrm{C}=\mathrm{O}}$ donor-acceptor interaction (Figure $\left.4 \mathrm{~d}\right)$. Indeed, a strong, negative correlation $\left(\mathrm{R}^{2}=\right.$ 0.90) exists, which is consistent with electron-withdrawing groups weakening a $\pi_{\mathrm{C}=\mathrm{C}} \leftrightarrow \pi^{*} \mathrm{C}=\mathrm{O}$ interaction.

a)

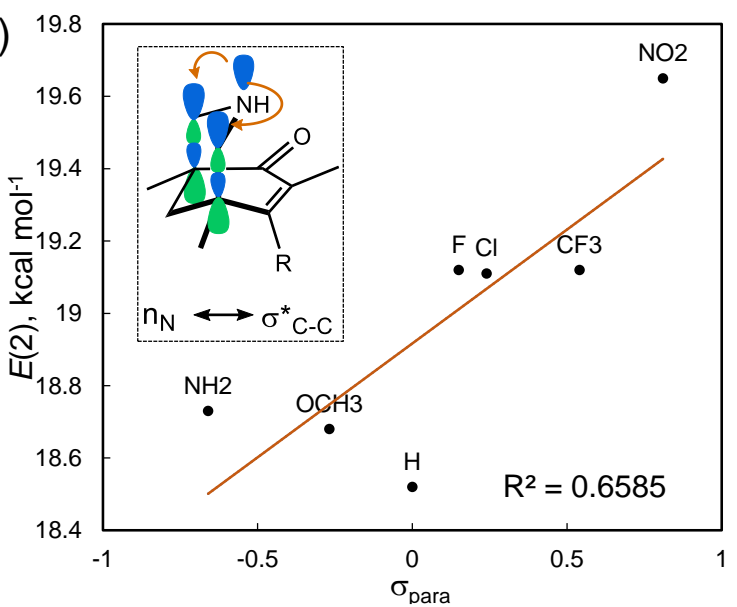

c)

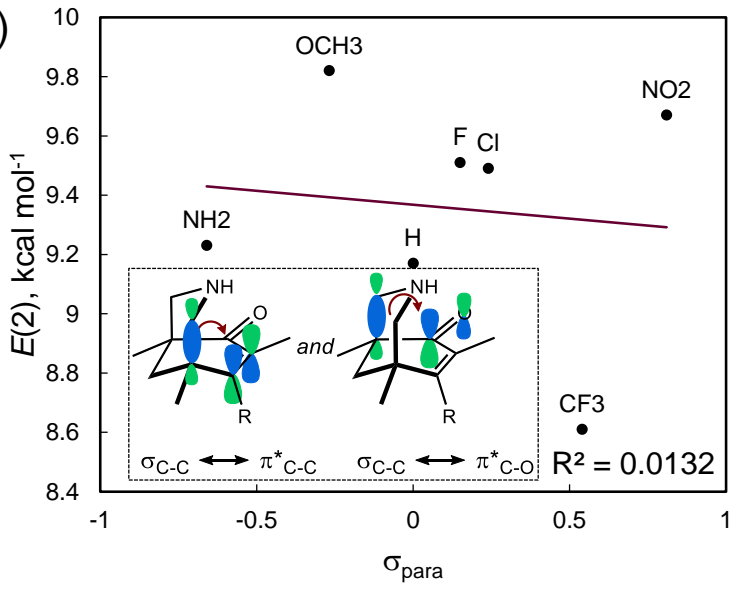

b)

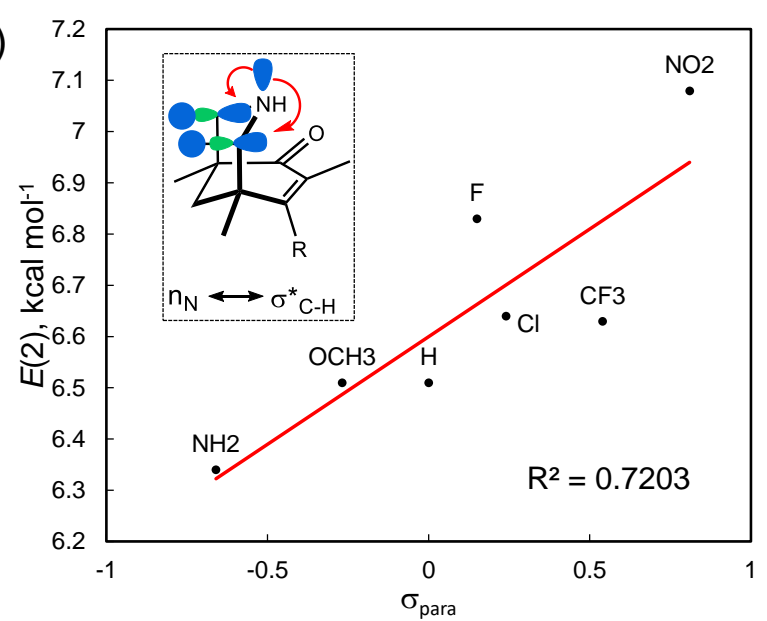

d)

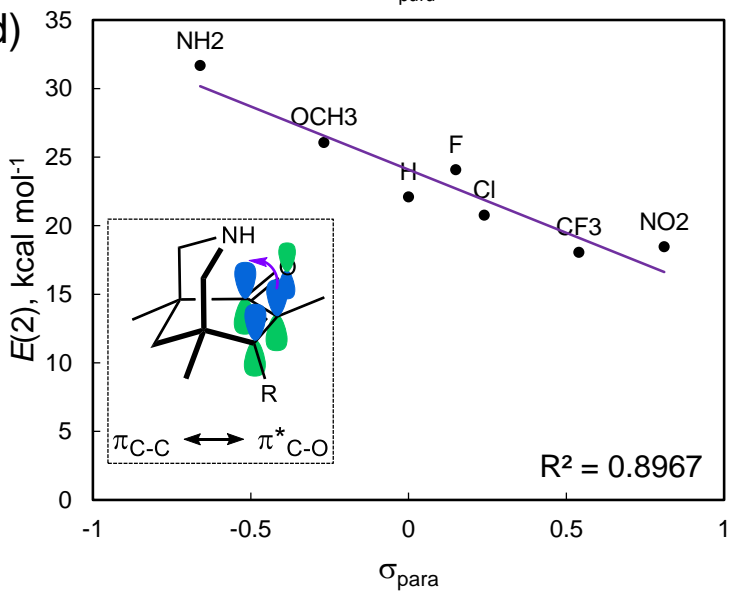

Figure 4. Correlations of second-order perturbation NBO energies, $E(2)$, and Hammett parameter, $\sigma_{\text {para }}$ (proxy for electron-donating and -withdrawing ability of substituent, R). Shown are correlations of $E(2)$ values associated with a) sum of negative hyperconjugation interactions of $\mathrm{n}_{\mathrm{N}} \leftrightarrow \sigma^{*}{ }_{\mathrm{C}-\mathrm{C}}$ of adjacent C-C sigma bonds, b) sum negative hyperconjugation interactions of $\mathrm{n}_{\mathrm{N}}$ 
$\leftrightarrow \sigma^{*}{ }_{\mathrm{C}-\mathrm{H}}$ of adjacent $\mathrm{C}-\mathrm{H}$ sigma bonds, c) sum of $\sigma_{\mathrm{C}-\mathrm{C}} \leftrightarrow \pi^{*}{ }_{\mathrm{C}=\mathrm{C}}$ and $\sigma_{\mathrm{C}-\mathrm{C}} \leftrightarrow \pi^{*}{ }_{\mathrm{C}=\mathrm{O}}$ interactions, and d) $\pi_{\mathrm{C}=\mathrm{C}} \leftrightarrow \pi^{*}{ }_{\mathrm{C}=\mathrm{O}}$ interaction.

In Figure 4a, we observe a slight correlation with the sum of $E(2)$ values and $\pi$ electrondonating and -withdrawing ability, but are the $\mathrm{n}_{\mathrm{N}} \leftrightarrow \sigma^{*}{ }_{\mathrm{C}-\mathrm{C}}$ interactions with adjacent $\mathrm{C}-\mathrm{C} \sigma$ bonds evenly distributed between the two bonds? The range of $E(2)$ values in Figure 5 a for the component associated with the "proximal" donor-acceptor interaction (proximal to the R group) is effectively the same as that in $5 \mathrm{~b}$ for the "distal" interaction (distal to the R group), $\sim 0.7$ $\mathrm{kcal} / \mathrm{mol}$, consistent with an evenly delocalized nitrogen lone pair into (seemingly indistinguishable) empty antibonding orbitals. However, we find that there is no correlation $\left(\mathrm{R}^{2}\right.$ $=0.14$ ) with respect to the proximal $\mathrm{n}_{\mathrm{N}} \leftrightarrow \sigma^{*}{ }_{\mathrm{C}-\mathrm{C}} E(2)$ energy (Figure 5a) and an excellent correlation $\left(\mathrm{R}^{2}=0.98\right)$ with respect to the distal $\mathrm{n}_{\mathrm{N}} \leftrightarrow \sigma^{*}{ }_{\mathrm{C}-\mathrm{C}} E(2)$ energy (Figure $\left.5 \mathrm{~b}\right)$. The origin of this difference appears to be related to the other partner in the hyperconjugation/conjugation array, i.e., the $\alpha, \beta$-unsaturated carbonyl. We observe an unequal distribution of $E(2)$ values for donation from $\mathrm{C}-\mathrm{C}$ bonds into adjacent $\pi^{*}$-antibonding orbitals, with greater $E(2)$ values for the distal interaction $\left(\sigma_{\mathrm{C}-\mathrm{C}} \leftrightarrow \pi^{*} \mathrm{C}=\mathrm{O}\right.$, Figure $\left.5 \mathrm{~d}\right)$ than the proximal interaction $\left(\sigma_{\mathrm{C}-\mathrm{C}} \leftrightarrow \pi^{*}{ }_{\mathrm{C}=\mathrm{C}}\right.$, Figure 5c), consistent with $\pi^{*} \mathrm{C}=\mathrm{O}$ being a better acceptor than is $\pi^{*} \mathrm{C}=\mathrm{C}$. In short, the carbonyl modulates communication between the substituent and the distal $\mathrm{C}-\mathrm{C}$ bond. 
a)

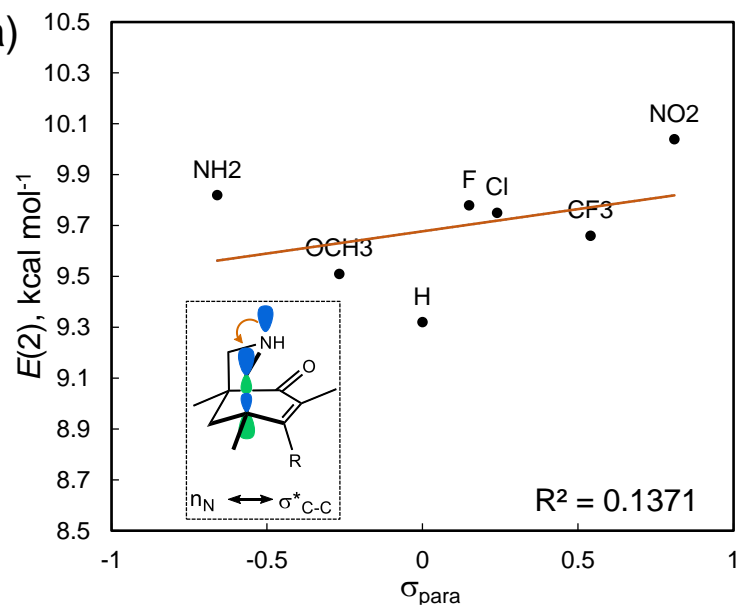

C)

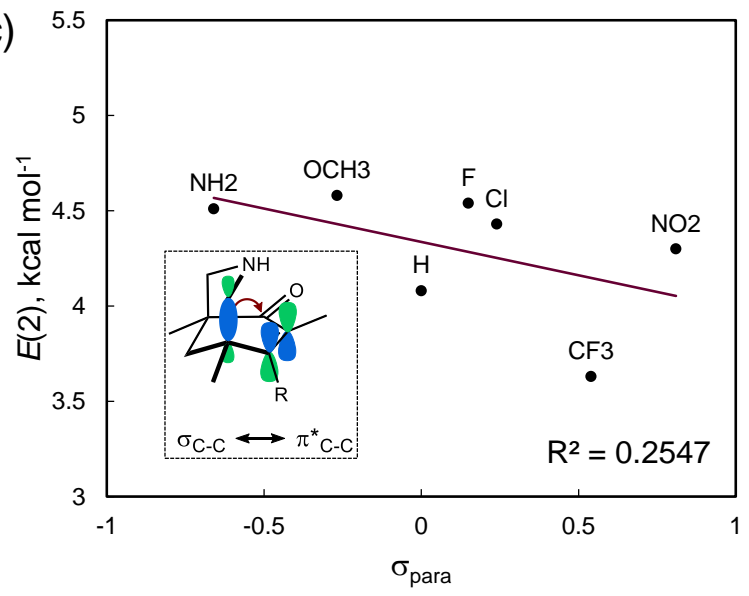

b)
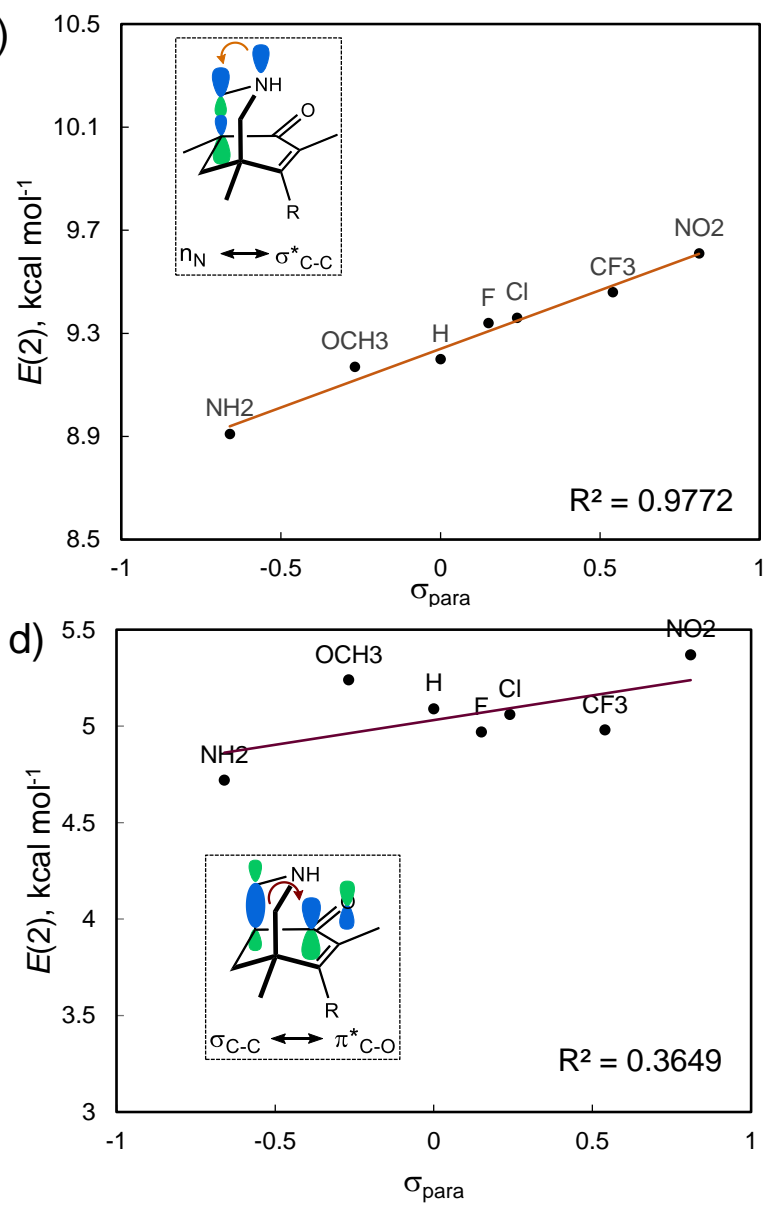

Figure 5. Correlations of second-order perturbation NBO energies, $E(2)$, and Hammett parameter, $\sigma_{\text {para }}$ (a measure for electron-donating/-withdrawing ability of substituent, R). Shown are correlations of $E(2)$ values associated with a) $n_{N} \leftrightarrow \sigma^{*}{ }_{C-C}$ interaction of proximal C-C sigma bonds, b) $n_{N} \leftrightarrow \sigma^{*}{ }_{C-C}$ interaction of distal C-C sigma bonds, c) $\sigma_{\mathrm{C}-\mathrm{C}} \leftrightarrow \pi^{*} \mathrm{C}=\mathrm{C}$ interaction of proximal C-C sigma bond with $\pi^{*} \mathrm{C}=\mathrm{C}$ of enone d) $\pi_{\mathrm{C}=\mathrm{C}} \leftrightarrow \pi^{*} \mathrm{C}=\mathrm{O}$ interaction of distal C-C sigma bond with $\pi^{*} \mathrm{C}=\mathrm{O}$ of enone. 
To isolate key stereoelectronic effects, thermochemical data derived experimentally or computationally are often used in hypothetical reactions (e.g., isogyric, isodesmic, hypohomodesmotic, homodesmotic, hyperhomodesmotic) ${ }^{84}$ These reactions can provide useful information for deducing how strong a delocalizing stereoelectronic effect is, but they are challenging to implement in practice because achieving an "ideal" reaction requires careful balancing of changes in bond type, charge, hybridization, and steric effects. ${ }^{20}$ Often, changing a portion of a molecule to probe an effect introduces additional, sometimes undesirable interactions. Figure 6, for example, shows simple isodesmic reactions that switch the lone pair position from equatorial to axial (i.e., $\mathbf{4} \rightarrow \mathbf{4}^{\prime}$ and $\mathbf{5} \rightarrow \mathbf{5}^{\prime} ; \mathbf{4}^{\prime}$ and $\mathbf{5}^{\prime}$ ' are examples of concave

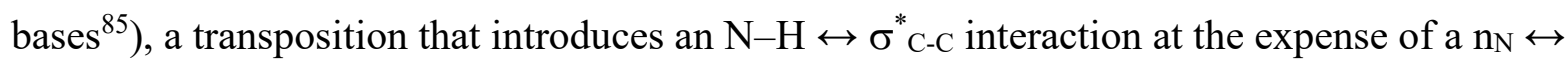
$\sigma_{\mathrm{C}-\mathrm{C}}^{*}$ interaction-for both $\mathbf{4}$ and $\mathbf{5}$, the reaction is uphill by at least $1 \mathrm{kcal} \mathrm{mol}^{-1}$. Though this hypothetical reaction seems like a reasonable method for acquiring a qualitative measure of the energetic stabilization due to nitrogen lone pair hyperconjugation, by switching the lone pair's position, we also introduce possible through-space effects between the $\mathrm{n}_{\mathrm{N}}$ and the $\pi$-system (vide supra). Like any isodesmic reaction, this one has flaws: the best we can do is asymptotically approach the 'ideal' isodesmic reaction. 


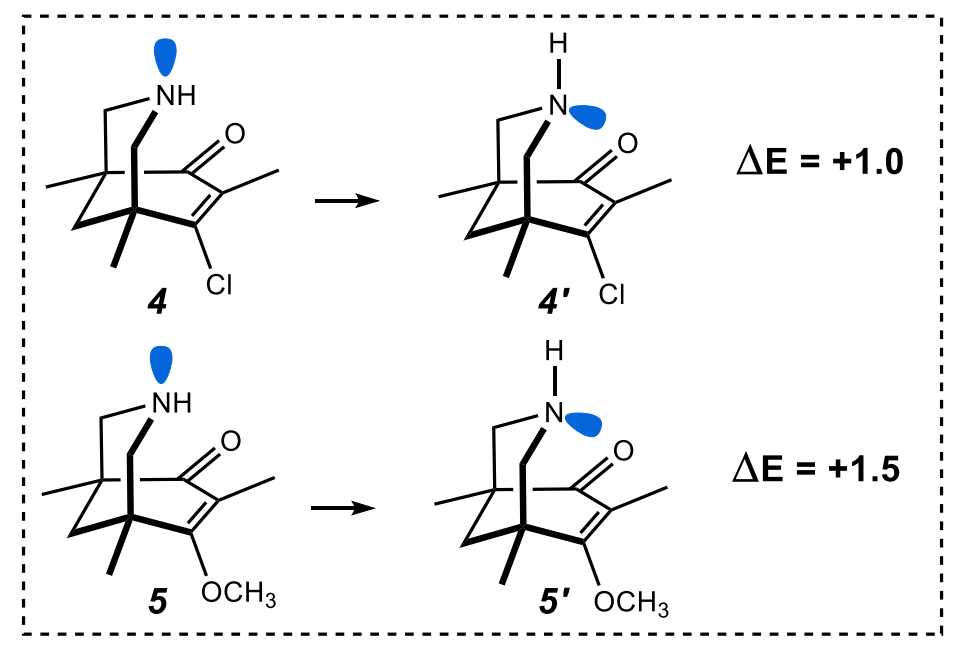

Figure 6. Isodesmic reaction. Amine inversion: lone pair from equatorial (4 and 5) to axial position (4' and $\mathbf{5}^{\prime}$ ).

Other possible isodesmic reactions are shown in Figure 7. Replacing the amine with a methylene $\left(\mathrm{CH}_{2}\right)$ group would, in principle, take away any energetic stabilization from the amine lone pair participating in hyperconjugation/conjugation. To keep each side balanced, we added a cyclohexane chair and a piperidine chair on the left and right-hand side, respectively. The $\Delta \mathrm{E}$ for this reaction is, roughly, a measure of the difference between having amine lone pair hyperconjugative stabilization and $\sigma_{\mathrm{C}-\mathrm{H}} \leftrightarrow \sigma^{*}{ }_{\mathrm{C}-\mathrm{C}}$ stabilization from a methylene group, but note that through-space interactions between the $\pi$-system and $\mathrm{N}-\mathrm{H}$ versus $\mathrm{C}-\mathrm{H}$ bonds are also present (although hopefully not significantly different). Taken together, the reactions shown in Figures 6 and $7 \mathrm{a}$ indicate that the net stabilization energy imparted by $n_{N} \leftrightarrow \sigma^{*}{ }_{\text {-C }}$ interactions in the context of other intermolecular interactions is likely on the order of $1 \mathrm{kcal} \mathrm{mol}^{-1}$ for both 4 and $\mathbf{5}$, and slightly more so for the latter. 

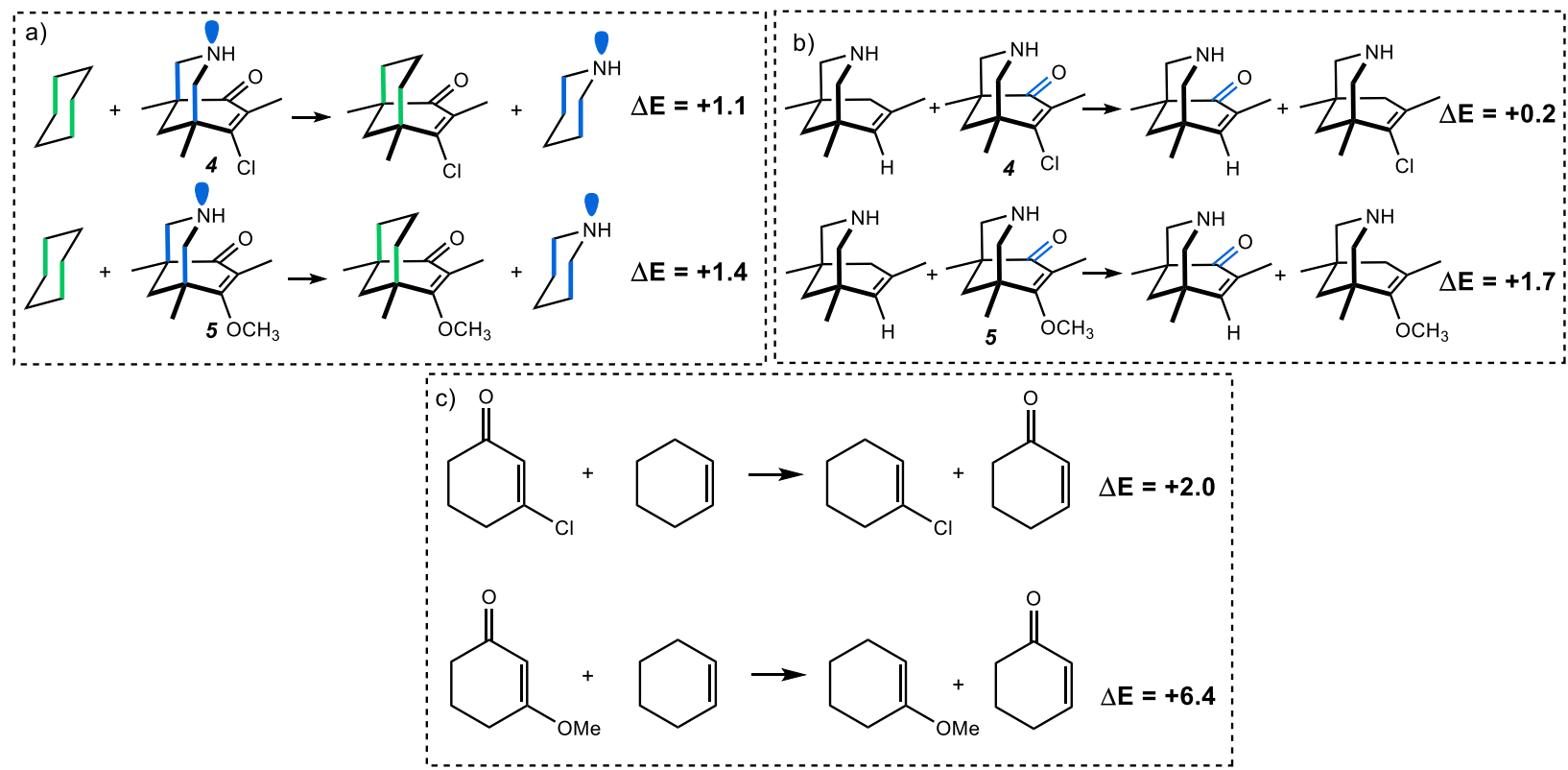

Figure 7. Isodesmic reactions. a) difference in stabilization energy due to amine lone pair versus C-H hyperconjugation into $\sigma^{*}$-C antibonding orbital for $\mathbf{4}$ and $\mathbf{5}$. B and c) "deletion" of ketone functionality.

Is there an isodesmic reaction that measures the stabilization energy associated with the carbonyl group of the enone? Figure 7b shows that "deleting" the carbonyl group in 4 costs almost nothing $\left(+0.2 \mathrm{kcal} \mathrm{mol}^{-1}\right)$, while “deleting” the carbonyl group in $\mathbf{5}$ imposes a $1.7 \mathrm{kcal}$ $\mathrm{mol}^{-1}$ energetic penalty, a reasonable reflection of the energetic stabilization due to a donor group at the $\beta$ position of a carbonyl group. Contrasting these results to those in Figure $7 \mathrm{c}$ suggests that the amine in the bicyclic molecule attenuates the cost imposed by ketone "deletion", consistent with the amine reducing the electrophilicity of the carbonyl group via the through-bond interaction described above, i.e., without the amine present, the communication between the $\mathrm{Cl} / \mathrm{OCH}_{3}$ and the carbonyl group through the intervening $\pi$-bond is stronger and the penalty for 
losing that communication is larger (see SI Figure S4 for additional computed isodesmic reactions).

A Closer Look-Trends in Basicity and Hydration Propensity

That the ketone oxygen is more basic in $\mathbf{5}$ than in $\mathbf{4}$ and more sensitive to the substituent at the C6 position is corroborated by the data in Figures 2 and 3. This result is not surprising. Changes to amine basicity were less obvious at the outset, however. Though changes to amine basicity with substituents at the carbonyl $\beta$-position are smaller than those to the carbonyl itself, they are substantial (cf. Figures 2-4). As the electron-withdrawing ability of the C6 substituent increases, the amount of through-bond lone pair delocalization increases (approximated with $E(2)$ in $\mathrm{kcal} \mathrm{mol}^{-1}$ ), and the amine basicity is curtailed (Figure 8).

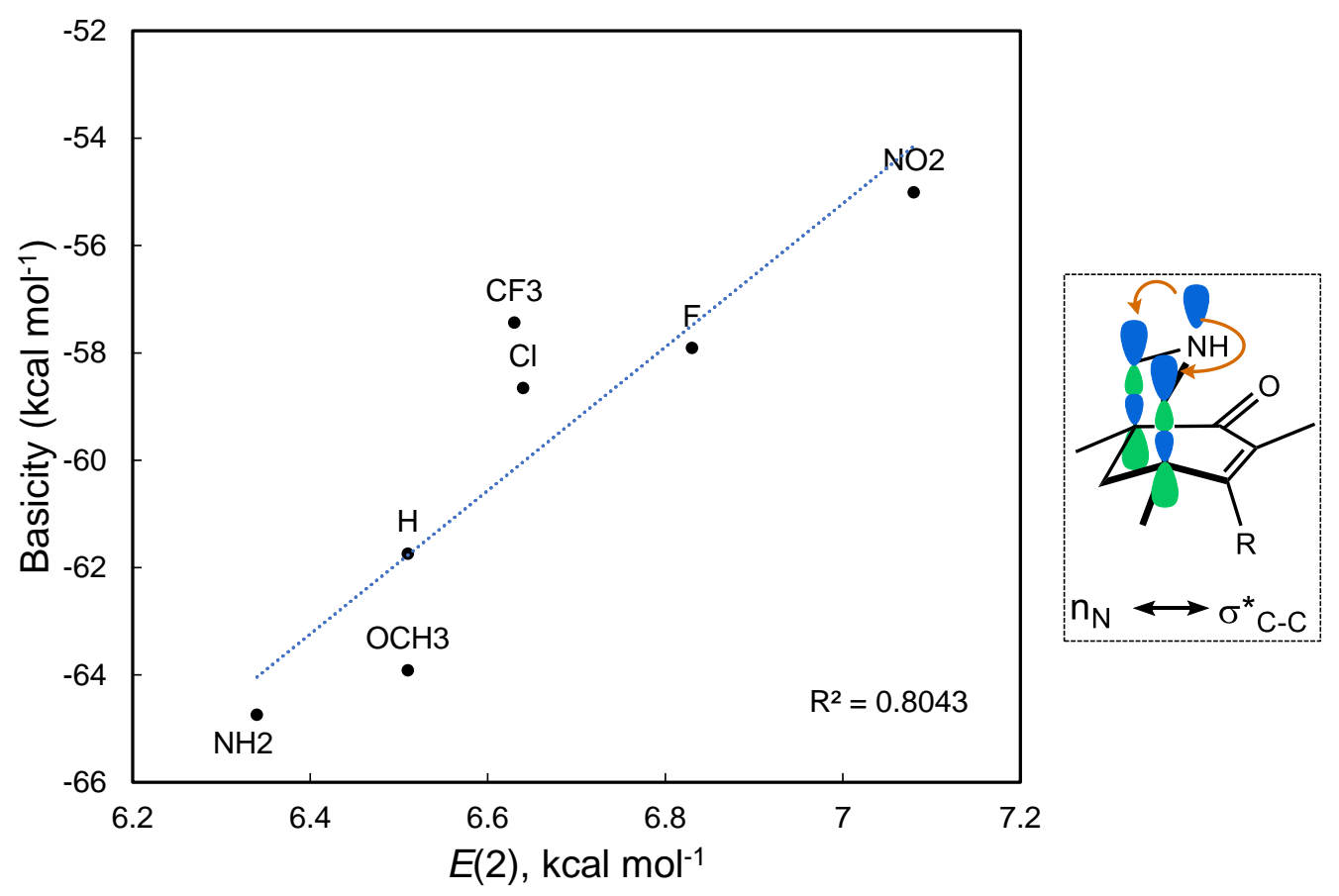


Figure 8. Relation of amine basicity to sum of $E(2) \mathrm{NBO}$ energies from $\mathrm{n}_{\mathrm{N}} \leftrightarrow \sigma^{*}{ }_{\mathrm{C}-\mathrm{C}}$ interaction.

An increased propensity for carbonyl protonation in $\mathbf{5}$ versus $\mathbf{4}$ should lead to increased electrophilicity, more facile attack by water, and more rapid hydrolysis (Scheme 3). But might an increased amine basicity have a similar effect? Covalent hydration propensities $\left(\ln K_{\text {hyd }}\right)$ were computed for C6-substituted systems with and without the amine protonated to estimate their reactivity towards water in the hydrolysis from 5 to 6 (Scheme 3 ). The more positive $\ln K_{\text {hyd, }}$, the greater the equilibrium lies toward the hydrate, suggesting a greater propensity to be attacked by water.

Scheme 3. Plausible mechanism from 5 to 1 via hydrolyzed product . $^{48}$

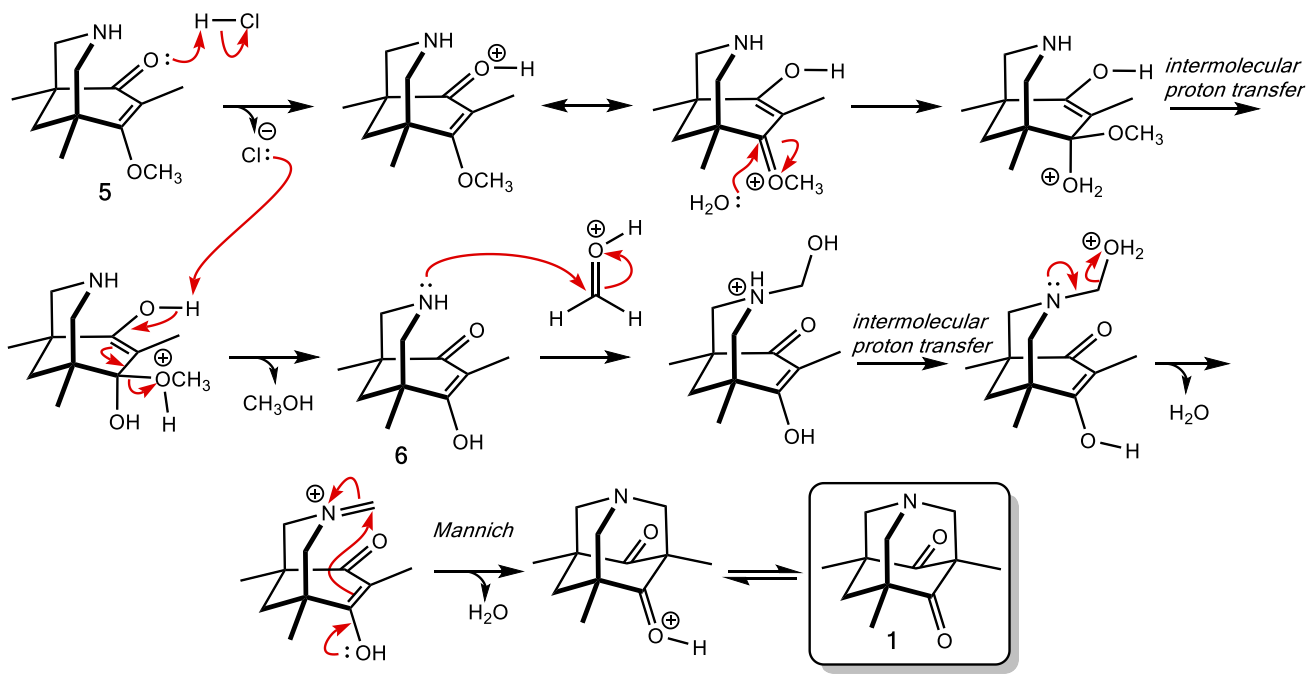

Three hydration sites were considered, for both neutral and $N$-protonated forms of $\mathbf{4}$ and 5 (a total of six hydration scenarios; see SI Figure S5 for details): (a) hydration at the carbonyl carbon, (b) hydration at C6 ( $\beta$-position) resulting from water addition from the "bottom" face 
(i.e., distal with respect to the amine), and (c) hydration at $\mathrm{C} 6$ resulting from water addition from the "top" face (i.e., proximal with respect to the amine). In all scenarios, $N$-protonated versions showed overall greater hydration propensities than their non-protonated counterparts. Electronwithdrawing groups at $\mathrm{C} 6$ also showed greater hydration propensities (e.g., Figure 9; see SI for other scenarios).

For 4 and 5, whether hydration is favored at the carbonyl carbon or the $\beta$-carbon depends on $\mathrm{C} 6$ substitution. In the case of $\mathbf{4}$, when the amine is protonated, hydration propensities at all possible sites are approximately equal $\left(\ln K_{\mathrm{hyd}} \sim 24\right)$. For $\mathbf{5}$, when the amine is protonated, hydration at the $\beta$-carbon resulting from "bottom" face attack by water (the face distal with respect to the amine) is most favored $\left(\ln K_{\text {hyd }}=23.5\right)$. Thus, we proposed a mechanism in Scheme 3 wherein water attacks the $\beta$-carbon from the "bottom" face ( $\ln K_{\text {hyd }}$ for "top" face water attack is 17.9). For this hydration scenario, $N$-protonation makes the hydration propensity for $4\left(\ln K_{\text {hyd }}\right.$ $=23.7)$ and $\mathbf{5}$ about equal $\left(\ln K_{\text {hyd }}=23.5\right.$; Figure 9$) !$ An equal susceptibility for water attack such as this is not present when the amines of $\mathbf{4}$ and $\mathbf{5}$ are unprotonated (see SI, Figure S8); in fact, in that case, $\mathbf{4}$ is more likely to be hydrated ( $\ln \mathrm{K}_{\text {hyd }}=10.9$ for $\mathbf{4}$ versus 7.2 for $\mathbf{5}$ ). In acid, however, the amines are likely protonated.

These hydration propensity results add yet another explanatory (albeit complex) layer to the mix: that is, despite hydration propensities for $\mathbf{4}$ and $\mathbf{5}$ being equal once their amines are protonated, 5's amine is more basic (Figure 8), hence more vulnerable to water attack. This result is consistent with the experimental observation that $\mathbf{5}$ is less kinetically stable under acidic conditions. Though we hesitate to single out any one property responsible for the stability of these molecules, the data is consistent with one overriding message: through-bond interactions modulate the reactivity of both the amine and enone components of $\mathbf{4}$ and $\mathbf{5}$. 


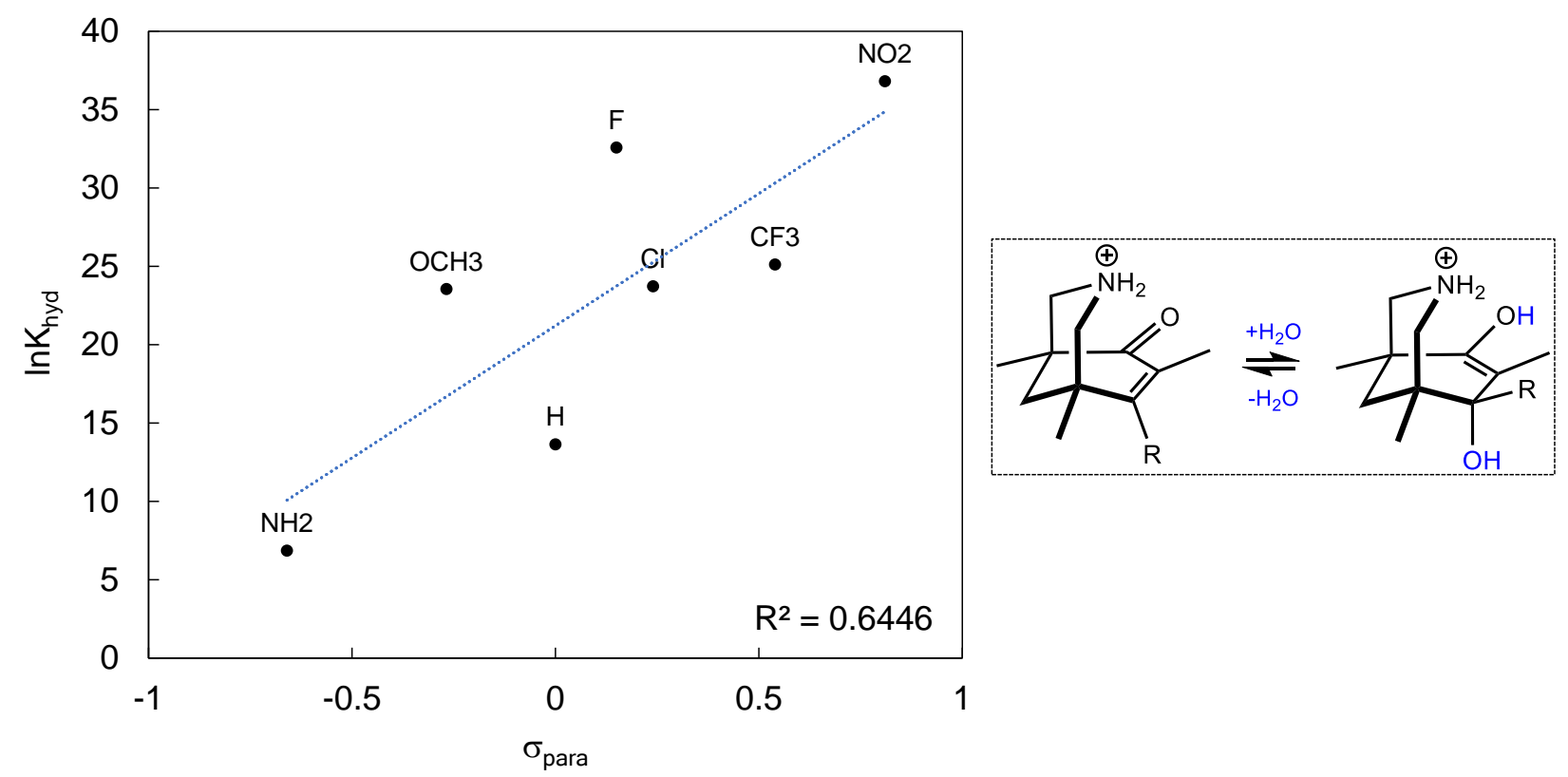

Figure 9. Hydration propensities ( $\left(\mathrm{ln}_{\mathrm{hyd}}\right)$ decrease as electron-withdrawing ability of $\mathrm{R}$ increases. The greater $\ln \mathrm{K}_{\text {hyd }}$ the greater the energetic driving force to be hydrated by $\mathrm{H}_{2} \mathrm{O}$.

THE UPSHOT-A MODEL FOR THROUGH-BOND EFFECTS IN $3-$ AZABICYCLO[3.3.1]NONANES

So, why is $\mathbf{5}$ unstable in acidic solution and $\mathbf{4}$ stable? First, it should be emphasized, as was stressed in a recent review article by Alabugin et al., ${ }^{52}$ that "molecular stability always depends on multiple factors. Singling one out of many can be misleading - unless there is a reason." In this study, we singled out particular stereoelectronic interactions to decide if the strengths of these interactions correlated with the $\pi$-donating/withdrawing ability of the substituent at C6. While correlation does not equate to causation, ${ }^{86}$ the data gathered up to this point allow us to suggest a working model rooted in through-bond effects. This model hinges on the reactivity of both the more-basic amine nitrogen and the less-basic ketone oxygen, which 
communicate with each other through intermediary $\sigma$-bonds. This study highlights the importance of remote through-bond effects (specifically, negative hyperconjugation/conjugation) in organic reactivity, an area still rich in opportunity for discovery. To not overlook (or underestimate) the importance of such interactions could make the difference between achieving a working model for explaining divergent reactivity of two (or more) molecules and being left in the dark.

\section{ASSOCIATED CONTENT}

Supporting Information. Supporting Data and Figures; Energies and Frequencies of Computed Structures (PDF).

A data set collection of computational results, including geometries and structure coordinates, is available in the ioChem-BD repository and can be accessed via https://doi.org/10.19061/iochem$\underline{\text { bd-6-120 }}$

\section{AUTHOR INFORMATION}

\section{Corresponding Author}

Dean J. Tantillo—Department of Chemistry, University of California_-Davis, CA 95616; email: djtantillo@ucdavis.edu; ORCID: https://orcid.org/0000-0002-2992-8844

\section{Authors}

Croix J. Laconsay_Department of Chemistry, University of California—Davis, CA 95616;

ORCID: https://orcid.org/0000-0002-9244-1318

Tyler C. Rho-Department of Chemistry, University of California-Davis, CA 95616 


\section{ACKNOWLEDGEMENTS}

Support from the US National Science Foundation (CHE-1856416 and XSEDE) is gratefully acknowledged.

\section{Author Contributions}

These authors contributed equally.

\section{REFERENCES}

(1) Young, T. Dr. No; United Artists: United Kingdom, 1962.

(2) Hoffmann, R. Interaction of Orbitals through Space and through Bonds. Acc. Chem. Res. 1971, 4, 1-9.

(3) Paddon-Row, M. N. Some Aspects of Orbital Interactions through Bonds: Physical and Chemical Consequences. Acc. Chem. Res. 1982, 15, 245.

(4) Hoffmann, R.; Imamura, A.; Hehre, W. J. Benzynes, Dehydroconjugated Molecules, and the Interaction of Orbitals Separated by a Number of Intervening Bonds. J. Am. Chem. Soc. 1968, 90, 1499-1509.

(5) Baker, A. D. Photoelectron Spectroscopy. Acc. Chem. Res. 1970, 3, 17-25.

(6) Dougherty, D. A.; Brint, P.; McGlynn, S. P. Photoelectron Spectroscopy of Carbonyls. Lone-Pair Interactions in .Alpha.-, .Beta.-, .Gamma.-, and .Delta.-Dicarbonyls. J. Am. Chem. Soc. 1978, 100, 5597-5603.

(7) Pasman, P.; Rob, F.; Verhoeven, J. W. Intramolecular Charge-Transfer Absorption and Emission Resulting from through-Bond Interaction in Bichromophoric Molecules. J. Am. Chem. Soc. 1982, 104, 5127-5133.

(8) Martin, H.; Mayer, B. Proximity Effects in Organic Chemistry-The Photoelectron Spectroscopic Investigation of Non-Bonding and Transannular Interactions. Angew. Chem. Int. Ed. 1983, 22, 283-314.

(9) Rademacher, P. Transannular Interactions in Difunctional Medium Rings - Modelling Bimolecular Reactions. Chem. Soc. Rev. 1995, 24, 143-150.

(10) Gleiter, R.; Kobayashi, M.; Kuthan, J. The N-Orbital Sequence in 1,3 Diazaadamantane. Tetrahedron 1976, 32, 2775-2781.

(11) Alabugin, I. V. Stereoelectronic Effects: A Bridge Between Structure and Reactivity, 1st ed.; John Wiley \& Sons, Ltd: Hoboken, NJ, 2016.

(12) Gonikberg, E. M.; Le Noble, W. J. Face Selection in Reactions of 5,7-Diazaadamantan-2- 
One Derivatives: Mutual Influence of Remote Substituents. J. Org. Chem. 1995, 60, 7751-7755.

(13) Gonikberg, E. M.; Picart, F.; Le Noble, W. J. Enhanced Stereoselectivity in the Capture of a 5-Substituted 2-Adamantyl Radical by Substitution of C-5 by Negative Boron. J. Org. Chem. 1996, 61, 9588-9590.

(14) Kaselj, M.; Gonikberg, E. M.; Le Noble, W. J. Effect of Remote Substitution on Face Selection in Addition Reactions of Nor- And Homoadamantan-9-Ones and of Several Analogues. J. Org. Chem. 1998, 63, 3218-3223.

(15) Adcock, W.; Trout, N. A. Nature of the Electronic Factor Governing Diastereofacial Selectivity in Some Reactions of Rigid Saturated Model Substrates. Chem. Rev. 1999, 99, 1415-1435.

(16) Lampkins, A. J.; Li, Y.; Al Abbas, A.; Abboud, K. A.; Ghiviriga, I.; Castellano, R. K. Assessable Consequences of Through-Bond Donor-Acceptor Interactions in $\beta$ Aminoketones. Chem. Eur. J. 2008, 14, 1452-1463.

(17) Dekkers, A. W. J. D.; Verhoeven'f, J. W.; Speckamp, W. N. On the Nature of SigmaCoupled Transitions. Through-Bond Interactions in 1-Aza-Adamantane Derivatives. Tetrahedron 1973, 29, 1691-1696.

(18) Sasaki, T.; Eguchi, S.; Kiriyama, T.; Sakito, Y.; Kato, H. Photoelectron Spectroscopic Evidence Concerning Ground-State Through-Sigma-Bond Interaction in the 1,3-DiazaAdamantan-6-One System. J. Chem. Soc. Chem. Commun. 1974, 725-726.

(19) Bishop, R.; Lee, G.-H. Detection of Non-Conjugative Interactions in Rigid Cyclic Molecules by Using Carbon-13 N.M.R. Shift Values. Aust. J. Chem. 1987, 40, 249-255.

(20) Alabugin, I. V.; dos Passos Gomes, G.; Abdo, M. A. Hyperconjugation. Wiley Interdiscip. Rev. Comput. Mol. Sci. 2018, e1389.

(21) Wu, J. I.-C.; Schleyer, P. von R. Hyperconjugation in Hydrocarbons: Not Just a "Mild Sort of Conjugation." Pure Appl. Chem. 2013, 85, 921-940.

(22) Risch, N.; Saak, W. Synthesis of Specifically Substituted 1-Azaadamantanes. Angew. Chem. Int. Ed. 1982, 21, 923-924.

(23) Risch, N. 1-Aza-Adamantanes from Phloroglucinols. J. Chem. Soc. Chem. Commun. 1983, 532-533.

(24) Risch, N.; Langhals, M.; Hohberg, T. Triple (Grob) Fragmentation. Retro-Mannich Reactions of 1-Aza-Adamantane Derivatives. Tetrahedron Lett. 1991, 32, 4465-4468.

(25) Worrell, C.; Verhoeven, J. W.; Speckamp, W. N. Through-Bond Interaction in 1-AzaAdamantane Derivatives. Tetrahedron 1974, 30, 3525-3531.

(26) Morishima, I.; Okada, K.; Yonezawa, T.; Goto, K. Studies on the Carbon-13 Contact Shifts of a Sigma-Electron System. Conformational Dependence of Carbon-13 Contact Shifts in Six-Membered Rings. J. Am. Chem. Soc. 1971, 93, 3922-3926.

Hoffmann, R. Molecular Beauty. In Roald Hoffmann on the Philosophy, Art, and Science 
of Chemistry; Oxford University Press, Inc.: New York, 2012; pp 272-292.

(28) Risch, N.; Billerbeck, U.; Meyer-Roscher, B. Azabicyclononane Durch Fragmentierung, II. Regiospezifische (Grob-)Fragmentierung Zur Darstellung von 3-Azabicyclononanen. Chem. Ber. 1993, 126, 1137-1140.

(29) Ruenitz, P. C.; Mokler, Corwin, M. Analogues of Sparteine. 5. Antiarrhythmic Activity of Selected N,N'-Disubstituted Bispidines. J. Med. Chem. 1977, 20, 1668-1671.

(30) Thompson, M. D.; Smith, G. S.; Berlin, K. D.; Holt, E. M.; Scherlag, B. J.; Van Der Helm, D.; Muchmore, S. W.; Fidelis, K. A. Synthesis and Antiarrhythmic Properties of Novel 3-Selena-7-Azabicyclo[3.3.1]Nonanes and Derivatives Single-Crystal X-Ray Diffraction Analysis of 7-Benzyl-3-Selena-7-Azabicyclo[3.3.1]Nonan-9-One and 7Benzyl-3-Selena-7-Azabicyclo[3.3.1]Nonane Hydroperchlo. J. Med. Chem. 1987, 30, 780-788.

(31) Marshall, L.; Parris, K.; Rebek, J.; Luis, S. V.; Burguete, M. I. A New Class of Chelating Agents. J. Am. Chem. Soc. 1988, 110, 5192-5193.

(32) Rebek, J. Molecular Recognition with Model Systems. Angew. Chem. Int. Ed. 1990, 29, 245-255.

(33) Kyburz, R.; Schöpp, E.; Bick, I. R. C.; Hesse, M. 258. Sorellin Und Hobartin, Zwei Neue Indolalkaloide Am Aristoteliu Peduncdaris (LABILL.) HOOK. F. Helv. Chim. Acta 1979, 62, 2539-2546.

(34) Argade, M. D.; Straub, C. J.; Rusali, L. E.; Santarsiero, B. D.; Riley, A. P. Synthesis of Aristoquinoline Enantiomers and Their Evaluation at the $\alpha 3 \beta 4$ Nicotinic Acetylcholine Receptor. Org. Lett. 2021, 23, 7693-7697.

(35) Eschenmoser, A.; Frey, A. Über Die Spaltung Des Mesylesters von 2-Methyl-2oxymethyl-cyclopentanon Mit Basen. Helv. Chim. Acta 1952, 35, 1660-1666.

(36) Grob, C. A.; Schiess, P. W. Heterolytic Fragmentation. A Class of Organic Reactions. Angew. Chem. Int. Ed. 1967, 6, 1-106.

(37) Weyerstahl, P.; Marschall, H. Fragmentation Reactions. In Comprehensive Organic Synthesis; 1991; pp 1041-1070.

(38) Prantz, K.; Mulzer, J. Synthetic Applications of the Carbonyl Generating Grob Fragmentation. Chem. Rev. 2010, 110, 3741-3766.

(39) Drahl, M. A.; Manpadi, M.; Williams, L. J. C-C Fragmentation: Origins and Recent Applications. Angew. Chem. Int. Ed. 2013, 52, 11222-11251.

(40) Hoang, T. T.; Dudley, G. B.; Williams, L. J. Fragmentation Reactions. In Comprehensive Organic Synthesis: Second Edition; Elsevier Ltd., 2014; Vol. 6, pp 842-860.

(41) Yang, J.; Hoang, T. T.; Dudley, G. B. Alkynogenic Fragmentation. Org. Chem. Front. 2019, 6, 2560-2569.

(42) Donald, J. R.; Unsworth, W. P. Ring-Expansion Reactions in the Synthesis of Macrocycles and Medium-Sized Rings. Chem. Eur. J. 2017, 23, 8780-8799. 
(43) Clarke, A. K.; Unsworth, W. P. A Happy Medium: The Synthesis of Medicinally Important Medium- Sized Rings via Ring Expansion. Chem. Sci. 2020, 11, 2876-2881.

(44) Maimone, T. J.; Shi, J.; Ashida, S.; Baran, P. S. Total Synthesis of Vinigrol. J. Am. Chem. Soc. 2009, 131, 17066-17067.

(45) Ferhati, X.; Salas-Cubero, M.; Garrido, P.; García-Sanmartín, J.; Guerreiro, A.; Avenoza, A.; Busto, J. H.; Peregrina, J. M.; Martínez, A.; Jiménez-Moreno, E.; et al. Bioorthogonal Self-Immolative Linker Based on Grob Fragmentation. Org. Lett. 2021, 23, 8580-8584.

(46) Laconsay, C. J.; Tsui, K. Y.; Tantillo, D. J. Tipping the Balance : Theoretical Interrogation of Divergent Extended Heterolytic Fragmentations. Chem. Sci. 2020, 11, 2231-2242.

(47) Schwarz, H. On the Usefulness of Useless Knowledge. Nat. Rev. Chem. 2017, 1, 1-3.

(48) Risch, N.; Langhals, M.; Mikosch, W.; Bögge, H.; Müller, A. Unusual Reorganization Reactions of 3-Azabicyclo[3.3.1]Nonanes. J. Am. Chem. Soc. 1991, 113, 9411-9412.

(49) Gleiter, R.; Stohrer, W.; Hoffmann, R. Orbital Theory of Heterolytic Fragmentation and Remote Effects on Nitrogen Inversion Equilibria. Helv. Chim. Acta 1972, 3, 893-906.

(50) Frisch, M. J.; Trucks, G. W.; Schlegel, H. B.; Scuseria, G. E. .; Robb, G. E.; Cheeseman, J. R.; Scalmani, G.; Barone, V.; Mennucci, B. .; Petersson, G. A.; Nakatsuji, H.; Caricato, M.; Li, X.; Hratchian, H. P. .; Izmaylov, A. F.; Bloino, J.; Zheng, G.; Sonnenberg, J. L.; Hada, M. .; Ehara, M.; Toyota, K.; Fukuda, R.; Hasegawa, J.; Ishida, M.; Nakajima, T. .; Honda, Y.; Kitao, O.; Nakai, H.; Vreven, T.; Montgomery, Jr., J. A. . P.; J. E.; Ogliaro, F.; Bearpark, M.; Heyd, J. J.; Brothers, E. . K.; K. N.; Staroverov, V. N.; Keith, T.;

Kobayashi, R.; Normand, J. . R.; K.; Rendell, A.; Burant, J. C.; Iyengar, S. S.; Tomasi, J. . C.; M.; Rega, N.; Millam, J. M.; Klene, M.; Knox, J. E.; Cross, J. B. .; et al. Gaussian 09, Revision D.01. Gaussian Inc. Wallingford, CT 2009.

(51) Zhao, Y.; Truhlar, D. G. The M06 Suite of Density Functionals for Main Group Thermochemistry, Thermochemical Kinetics, Noncovalent Interactions, Excited States, and Transition Elements: Two New Functionals and Systematic Testing of Four M06Class Functionals and 12 Other Function. Theor. Chem. Acc. 2008, 120, 215-241.

(52) Alabugin, I. V.; Kuhn, L.; Krivoshchapov, N. V.; Mehaffy, P.; Medvedev, M. G. Anomeric Effect, Hyperconjugation and Electrostatics: Lessons from Complexity in a Classic Stereoelectronic Phenomenon. Chem. Soc. Rev. 2021, 50, 10212-10252.

(53) Nam, S.; Cho, E.; Sim, E.; Burke, K. Explaining and Fixing DFT Failures for Torsional Barriers. J. Phys. Chem. Lett. 2021, 12, 2796-2804.

(54) Fukui, K. The Path of Chemical Reactions -- The IRC Approach. Acc. Chem. Res. 1981, 14, 363-368.

(55) Gonzalez, C.; Schlegel, H. B. Reaction Path Following In Mass-Weighted Internal Coordinates Cartesians and with Internal Coordinates without Mass-Weighting. J. Phys. Chem. 1990, 94, 5523-5527.

(56) Maeda, S.; Harabuchi, Y.; Ono, Y.; Taketsugu, T.; Morokuma, K. Intrinsic Reaction Coordinate: Calculation, Bifurcation, and Automated Search. Int. J. Quantum Chem. 
2015, 115, 258-269.

(57) Álvarez-Moreno, M.; De Graaf, C.; López, N.; Maseras, F.; Poblet, J. M.; Bo, C. Managing the Computational Chemistry Big Data Problem: The ioChem-BD Platform. $J$. Chem. Inf. Model. 2015, 55, 95-103.

(58) Prasad, S.; Tantillo, D. J. Substituent Effects on the Basicity of Patriscabrin A and Lettucenin A: Evolution Favors the Aromatic? ACS Omega 2021, 6, 29685-29691.

(59) Johnson, E. R.; Keinan, S.; Mori-Sánchez, P.; Contreras-García, J.; Cohen, A. J.; Yang, W. Revealing Noncovalent Interactions. J. Am. Chem. Soc. 2010, 132, 6498-6506.

(60) Lu, T.; Chen, F. Multiwfn: A Multifunctional Wavefunction Analyzer. J. Comput. Chem. 2012, 33, 580-592.

(61) Glendening, E. D.; Reed, A. E.; Carpenter, J. E.; Weinhold, F. NBO Version 3.1 Gaussian Inc. Pittsburgh 2003.

(62) Weinhold, F.; Landis, C. R.; Glendening, E. D. What Is NBO Analysis and How Is It Useful? Int. Rev. Phys. Chem. 2016, 35, 399-440.

(63) Wedler, H. B.; Palazzo, T. A.; Pemberton, R. P.; Hamann, C. S.; Kurth, M. J.; Tantillo, D. J. Predicting Hydration Propensities of Biologically Relevant $\alpha$-Ketoamides. Bioorganic Med. Chem. Lett. 2015, 25, 4153-4157.

(64) Buschmann, H.-J.; Dutkiewicz, E.; Knoche, W. Reversible Hydration of Carbonyl Compounds in Aqueous Solution - 2. the Kinetics of the Keto/Gem-Diol Transition. Ber. Bunsenges. Phys. Chem. 1982, 86, 129-134.

(65) Guthrie, J. P. Hydration of Carbonyl Compounds, an Analysis in Terms of Multidimensional Marcus Theory. J. Am. Chem. Soc. 2000, 122, 5529-5538.

(66) Wagner, J. P.; Schreiner, P. R. London Dispersion in Molecular Chemistry Reconsidering Steric Effects. Angew. Chem. Int. Ed. 2015, 54, 12274-12296.

(67) Peng, Q.; Duarte, F.; Paton, R. S. Computing Organic Stereoselectivity-from Concepts to Quantitative Calculations and Predictions. Chem. Soc. Rev. 2016, 45, 6093-6107.

(68) Maji, R.; Mallojjala, S. C.; Wheeler, S. E. Chiral Phosphoric Acid Catalysis: From Numbers to Insights. Chem. Soc. Rev. 2018, 47, 1142-1158.

(69) Morgenthaler, M.; Schweizer, E.; Hoffmann-Röder, A.; Benini, F.; Martin, R. E.; Jaeschke, G.; Wagner, B.; Fischer, H.; Bendels, S.; Zimmerli, D.; et al. Predicting and Tuning Physicochemical Properties in Lead Optimization: Amine Basicities. ChemMedChem 2007, 2, 1100-1115.

(70) Hammett, L. P. The Effect of Structure upon the Reactions of Organic Compounds. Benzene Derivatives. J. Am. Chem. Soc. 1937, 59, 96-103.

(71) Hansch, C.; Leo, A.; Taft, R. W. A Survey of Hammett Substituent Constants and Resonance and Field Parameters. Chem. Rev. 1991, 91, 165-195.

(72) Kardel, D.; Knoche, W.; Risch, N. Protonation and Gem-Diol Formation of 1- 
Azaadamantanones. J. Chem. Soc. Perkin Trans. 2 1993, 2, 1455-1459.

(73) Galasso, V. On the $\mathrm{n}(\mathrm{N}) / \mathrm{n}(\mathrm{O})$ Interaction in Adamantane Cages. J. Mol. Struct. THEOCHEM 2000, 528, 171-176.

(74) Lambert, J. B.; Ciro, S. M. The Interaction of $\pi$ Orbitals with a Carbocation over Three $\sigma$ Bonds. J. Org. Chem. 1996, 61, 1940-1945.

(75) Alabugin, I. V; Manoharan, M. Effect of Double-Hyperconjugation on the Apparent Donor Ability of $\sigma$-Bonds: Insights from the Relative Stability of $\delta$-Substituted Cyclohexyl Cations. J. Org. Chem. 2004, 69, 9011-9024.

(76) Grob, Cyril A, R. R. Inductive and Hyperconjugative Effects in the Solvolysis of 4Substituted Bicyclo[2.2.2]Oct-1-Yl p-Nitrobenzenesulfonates. Tetrahedron Lett. 1978, 663-666.

(77) Wu, J. I.; Fernández, I.; Mo, Y.; Von Ragué Schleyer, P. Why Cyclooctatetraene Is Highly Stabilized: The Importance of "Two-Way" (Double) Hyperconjugation. J. Chem. Theory Comput. 2012, 8, 1280-1287.

(78) Grob, C. A.; Rich, R. Polar Effects in the Solvolysis of 4-Substituted Bicyclo [2.2.2]Octyl P-Nitrobenzenesulfonates. Polar Effects. VII. Helv. Chim. Acta 1979, 62, 2793-2801.

(79) Adcock, W.; Krstic, A. R.; Duggan, P. J.; Shiner, V. J.; Coope, J.; Ensinger1, M. W. Through-Bond Transmission of Substituent Effects in the Bicyclo[2.2.2]Octane Ring System: Solvolysis of 4-Deuterioand 4-Metalloidal (M(CH3)3, M = Si, Ge, and Sn)Substituted Bicyclo[2.2.2]Oct-1-Yl p-Nitrobenzenesulfonates and Methanesulfonates. $J$. Am. Chem. Soc. 1990, 112, 3140-3145.

(80) Adcock, W.; Trout, N. A. Transmission of Polar Substituent Effects in the Adamantane Ring System As Monitored by 19F NMR: Hyperconjugation as a Stereoinductive Factor. J. Org. Chem. 1991, 56, 3229-3238.

(81) Lambert, J. B.; Salvador, L. A.; So, J. H. The .Gamma. and .Delta. Effects of Tin. Organometallics 2005, 12, 697-703.

(82) Vidhani, D. V.; Krafft, M. E.; Alabugin, I. V. Gold(I)-Catalyzed Allenyl Cope Rearrangement: Evolution from Asynchronicity to Trappable Intermediates Assisted by Stereoelectronic Switching. J. Am. Chem. Soc. 2016, 138, 2769-2779.

(83) Reed, A. E.; Curtiss, L. A.; Weinhold, F. Intermolecular Interactions from a Natural Bond Orbital, Donor-Acceptor Viewpoint. Chem. Rev. 1988, 88, 899-926.

(84) Wheeler, S. E.; Houk, K. N.; Schleyer, P. V. R.; Allen, W. D. A Hierarchy of Homodesmotic Reactions for Thermochemistry. J. Am. Chem. Soc. 2009, 131, 25472560 .

(85) Lüning, U. Concave Reagents: Syntheses of Macrobicyclic Pyridines. Liebigs Ann. Chem. 1987, 949-955.

(86) Johnson, S. R. The Trouble with QSAR (or How I Learned To Stop Worrying and Embrace Fallacy). J. Chem. Inf. Model. 2008, 48, 25-26. 